\title{
OpenVirtualObjects: An Open Set of Standardized and Validated 3D Household Objects for Virtual Reality-Based Research, Assessment, and Therapy
}

\author{
Johanne Tromp ${ }^{1,2 \star}$, Felix Klotzsche ${ }^{1,2}$, Stephan Krohn ${ }^{3}$, Mert Akbal ${ }^{1,4}$, Leonardo Pohl ${ }^{5}$, \\ Eva M. Quinque ${ }^{1,6}$, Julia Belger ${ }^{1,6}$, Arno Villringer ${ }^{1,2}$ and Michael Gaebler ${ }^{1,2 *}$ \\ ${ }^{1}$ Neurology Department, Max Planck Institute for Human Cognitive and Brain Sciences, Leipzig, Germany, \\ ${ }^{2}$ Humboldt-Universität zu Berlin, Faculty of Philosophy, Berlin School of Mind and Brain, MindBrainBody Institute, Berlin, \\ Germany, ${ }^{3}$ Department of Neurology, Charité-Universitätsmedizin Berlin, Berlin, Germany, ${ }^{4}$ Hochschule der Bildenden \\ Künste Saar, Saarbrücken, Germany, ${ }^{5}$ Freie Universität Berlin, Berlin, Germany, ${ }^{6}$ Clinic for Cognitive Neurology, University \\ Hospital Leipzig, Leipzig, Germany
}

OPEN ACCESS

Edited by:

Mar Gonzalez-Franco,

Microsoft Research, United States

Reviewed by:

Jacob Thorn,

École Polytechnique Fédérale de

Lausanne, Switzerland

Miguel A. Otaduy,

Rey Juan Carlos University, Spain

*Correspondence:

Johanne Tromp

trompjohanne@gmail.com

Michael Gaebler

gaebler@cbs.mpg.de

Specialty section:

This article was submitted to Virtual Reality and Human Behaviour,

a section of the journal

Frontiers in Virtual Reality

Received: 28 September 2020

Accepted: 23 November 2020

Published: 22 December 2020

Citation:

Tromp J, Klotzsche F, Krohn S,

Akbal M, Pohl L, Quinque EM,

Belger J, Villringer $A$ and Gaebler $M$ (2020) OpenVirtualObjects: An Open Set of Standardized and Validated $3 D$

Household Objects for Virtual Reality-Based Research, Assessment,

and Therapy.

Front. Virtual Real. 1:611091.

doi: 10.3389/frvir.2020.611091
Virtual reality (VR) technology provides clinicians, therapists, and researchers with new opportunities to observe, assess, and train behavior in realistic yet well-controlled environments. However, VR also comes with a number of challenges. For example, compared to more abstract experiments and tests on 2D computer screens, VR-based tasks are more complex to create, which can make it more expensive and time-consuming. One way to overcome these challenges is to create, standardize, and validate VR content and to make it openly available for researchers and clinicians. Here we introduce the OpenVirtualObjects (OVO), a set of 124 realistic 3D household objects that people encounter and use in their everyday lives. The objects were rated by 34 younger and 25 older adults for recognizability, familiarity, details (i.e., visual complexity), contact, and usage (i.e., frequency of usage in daily life). All participants also named and categorized the objects. We provide the data and the experiment- and analysis code online. With OVO, we hope to facilitate VR-based research and clinical applications. Easy and free availability of standardized and validated 3D objects can support systematic VR-based studies and the development of VR-based diagnostics and therapeutic tools.

Keywords: virtual reality, 3D objects database, stimuli, neuropsychology \& neurology, virtual medicine, 3D objects

\section{INTRODUCTION}

Virtual reality (VR) technology provides computer-generated content based on a user's movement in or interaction with a simulated environment to enable an immersive experience (Aukstakalnis and Blatner, 1992; Rizzo and Koenig, 2017). In non-immersive VR, users interact with 3D computer graphics on a 2D screen, while in immersive VR, people are fully "embedded" in the virtual environment through the use of stereoscopic head-mounted displays (HMDs) and body-tracking sensors.

For users, VR enables interactive, immersive experiences. For clinicians and researchers, VR provides new opportunities to observe, assess, and train behavior in realistic environments and with new measures, for example, to treat phobias (Morina et al., 2015), post-traumatic stress disorder 
(e.g., Rizzo et al., 2010), or to rehabilitate attention, memory, and motor and spatial skills [for an overview, see Rizzo and Koenig (2017)]. In a recent overview of VR-based solutions for the diagnostics and therapy of neurological diseases, Słyk et al. (2019) argue that although VR is still relatively understudied, it is making visible progress.

VR can provide tailored trainings for different patient groups or even individual patients in controlled environments that resemble the everyday life of patients. Classical diagnostics and therapies often lack this resemblance or only achieve it at high costs. For example, spatial memory (i.e., the capacity to recognize or recall relationships between objects in space) is often assessed with paper-and-pencil tests like the Rey-Osterrieth Complex Figure Test (ROCF), in which participants have to copy a complex line drawing and recall it from memory (Rey, 1941; Osterrieth, 1944). In fundamental research, the mechanisms of working memory are regularly investigated with tasks in which participants have to memorize abstract shapes presented on a computer screen (e.g., Brady et al., 2016). Although many classical paradigms rely on controlled, de-contextualized environments and stimuli, clinicians and researchers did develop realistic tasks and experiments to study the behavior of patients and participants in everyday life situations: In the cooking task, for example, patients are asked to prepare a dish in a real kitchen in the clinic, while the therapist continuously monitors their performance (e.g., Frisch et al., 2012). However, these tasks are costly and time-consuming, because the therapist has to be present at all times and the real-life environment has to be physically built or made available. In addition, they often lack the experimental control necessary for systematic and reproducible results, and they often do not allow for automatic (i.e., experimenter-independent) multi-dimensional data collection.

VR could bridge the gap between the laboratory (i.e., systematic and controlled environments and tasks) and everyday life (i.e., natural situations and behavior). It allows for the systematic delivery of realistic yet well-controlled stimuli, realtime performance feedback, and a safe testing or training environment. From a clinical perspective, patients can also independently practice skills in a controlled environment outside the clinic and ideally at home (Rizzo and Koenig, 2017). An example of a successful clinical VR application is VR exposure therapy (VRET), in which "in virtuo" exposure can lead to changing one's behavior in real-life situations (Morina et al., 2015; Howard, 2017). Furthermore, executive functions, the higherorder cognitive processes required for performing complex tasks, have been assessed in healthy individuals and patients using action planning tasks in a virtual supermarket (Josman et al., 2008). VR provides precise outcome measures (e.g., information about the location and position of the head or other body parts), interactive dynamic experimental paradigms (e.g., using controllers or other methods for interaction with the environment) as well as high-resolution stimuli that can be completely controlled and easily matched for certain properties (e.g., color, size). Using stimuli with heightened realism, it has been shown that people can store more real-world objects (e.g., bell, cookie) than abstract shapes (e.g., squares varying in color and orientation) in memory, and they do so with more detail (Brady et al., 2016). This suggests that working memory capacity has been systematically underestimated in studies that used simple stimuli about which we have no previous knowledge. In addition, VR has been used to study spatial memory (e.g., Koenig et al., 2011), and it can be combined with neurophysiological measures such as electroencephalography (e.g., Klotzsche et al., 2018; Tromp et al., 2018).

Although VR is a promising tool for research and clinical applications, it also has several disadvantages, and its use comes with several challenges. While the price of VR hardware (e.g., commercial HMDs like the Oculus Quest) has dropped substantially, it is still rather expensive and time-consuming to develop VR-based experiments and applications. There is little VR content freely available that is suitable for experimental research and clinical applications, and the content that is available commonly lacks the empirical validation warranted to ensure scientific rigor. For example, VR content that is designed for entertainment or educational purposes is often not suitable for research, because stimulus properties are not matched or the experience cannot be modified to fit specific research questions. In addition, after a VR-based experiment has been successfully designed, the additional dimensions of behavioral assessment increase the number and complexity of outcome measures-and thereby the "researcher degrees of freedom" (Simmons et al., 2011)_even more than in classical (computerized) tasks in the psychological and biomedical sciences (Ioannidis, 2005). This is especially problematic for the comparison of performance between different VR paradigms or therapies, since even if the task is the same, there are many aspects of the VR experience (e.g., the environment, the interaction) that can cause different outcomes. To resolve some of these issues, our group, in previous research, presented a new framework, "the VR-check." This framework can be used to aid systematic and comparative paradigm optimization in VR-based research on the basis of several dimensions; including cognitive domain specificity, ecological relevance, technical feasibility, user feasibility, user motivation, task adaptability, performance quantification, immersive capacities, training feasibility, and predictable pitfalls (Krohn et al., 2020).

To further advance the standardization of VR-based experiments and applications (e.g., for diagnostics and training) and to reduce the costs of VR-based research, we created OpenVirtualObjects (OVO). OVO is a freely available set of 124 realistic 3D household objects for VR-based research and clinical applications (e.g., diagnostics and rehabilitation). VR provides a unique opportunity to create assessments and trainings that are relevant to the real environments of people, while still maintaining full control over the stimuli (unlike in the real world). Therefore, we selected objects that naturally occur in a household setting. This could increase the amount of transfer from training to real life use of certain skills and it could provide a more realistic assessment of cognitive abilities. The objects in the database were rated by younger and older adults on the dimensions of recognizability, familiarity, details (i.e., visual complexity), contact, and usage (i.e., frequency of usage in daily life). Participants also categorized and named the objects. 
OVO complements existing databases with 3D objects: For example, bigBIRD (Singh et al., 2014) contains 100 high-quality objects and is mostly targeted at computer vision researchers, but it does not contain norms relevant for experimental and clinical use. Also, Popic et al. (2020) provided a database of 121 objects normed for name, familiarity and visual complexity. Finally, Peeters (2018) provides a standardized set of 147 3D objects (e.g., bike, table) normed by younger adults for name agreement, image agreement, familiarity, and visual complexity. Names and lexical characteristics of the names are also described. The OVO objects complement these data sets because they were specifically selected and rated for their appearance in everyday life (i.e., household environments), and they were rated on these properties by both younger and older adults. The selection of objects in OVO aimed to increase the personal relevance of the objects-to maximize the link between the VR-based experiments or applications and everyday life.

In this paper, we describe how the objects were created and validated. We provide descriptive statistics as well as information on the relations between the norms, for example to provide some information on how the less common norms of contact and usage (i.e., frequency of usage in daily) relate to each other and to the more standard norms used in this study. We also present the naming and category data for the objects. To give the reader an overview of the properties of OVO as compared to items in other databases, we compared the overlapping ratings of OVO with existing 2D (Adlington et al., 2009; Brodeur et al., 2010; MorenoMartínez and Montoro, 2012) and 3D object databases (Peeters, 2018; Popic et al., 2020). Our goal is to provide clinical and fundamental research community with a validated resource for their own VR-based applications as well as their intended sample.

\section{METHODS}

\section{Participants}

Thirty-four younger (19 females; mean age: $28 \pm 4$.6; range $20-38$ years) and 25 older adults (14 females; mean age: $70 \pm 5.0$; range 62-82 years) participated in the study. The data from 3 subjects was discarded due to red-green blindness, early termination of the experiment, or technical difficulties. All participants were native speakers of German, right-handed, and had normal or corrected-to-normal vision. They provided written informed consent and were paid for participation $(9 € / \mathrm{h})$. Ethical approval was granted by the ethics committee of the Psychology Dept. at the Humboldt-Universität zu Berlin.

\section{Materials}

As the basis, 124 copyright-free (CC-Zero) 3D objects were downloaded from open platforms (e.g., www.blendswap.com). Selection criteria were (1) inanimateness and (2) occurrence in household settings (based on the knowledge and experience of the authors who selected the objects). A professional visual artist (Mert Akbal) edited the objects using Blender version 2.79 (www.blender.org). The goal was to create a homogenous set of objects with $<20,000$ vertices each and with minimal differences in size and other salient features. Some object types (e.g., bottle, orange) appeared more than once (in different versions) in the

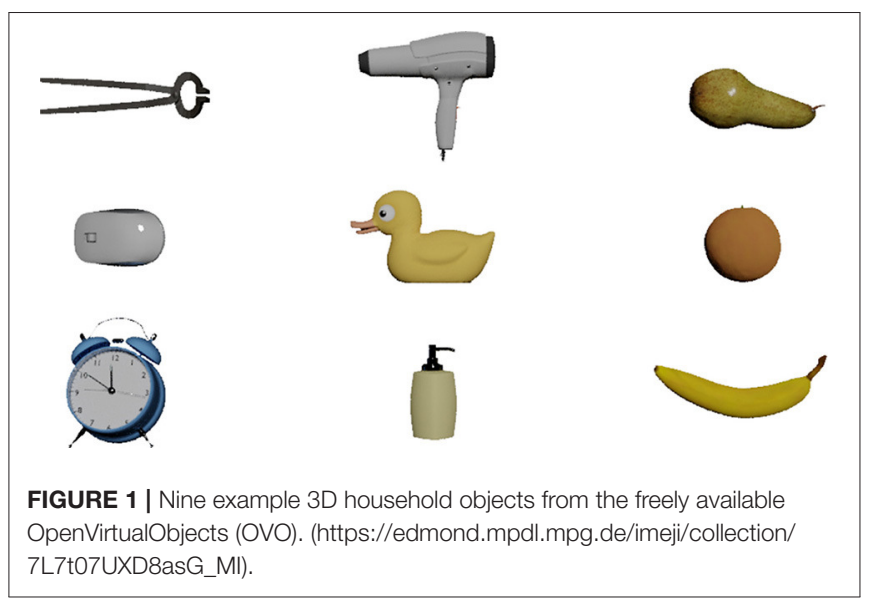

initial data set so that after the rating, the best version of the object could be selected. All objects (124) in OVO are freely available (in.blend and.fbx format) online. In an additional set, the objects were revised to facilitate usage in a real-time interactive VR experience by reducing the number of vertices. The high-poly versions have 10,000-20,000 and the "low-poly" versions have $<10,000$ vertices. One object (WineBottle1_MPI01) was removed from the analyses in this paper due to a scaling problem during the rating, but it is still in the objects folder. A list of the object names and a screenshot with all objects (.png) is also provided with the database (https://edmond.mpdl.mpg.de/imeji/ collection/7L7t07UXD8asG_MI). Figure 1 shows a selection of the objects.

\section{Apparatus}

The experiment was created in Unity version 2017.4.5 (www.unity.com), exported as a Linux standalone build, and run under Ubuntu 18.04 on desktop computers. Participants were seated $55 \mathrm{~cm}$ in front of a 22-inch computer screen (EIZO $\mathrm{S} 2202 \mathrm{~W})$ with a $1,680 \times 1,050$-pixel resolution. The Unity code for the rating experiment is available in the OVO database to facilitate the collection of additional ratings or to run rating studies for new objects.

\section{Procedure}

All ratings and names were collected in one experimental session. Before the experiment started, participant provided informed written consent. Participants were instructed that they would see several objects, which they should rate and name. The experiment consisted of five "scenes," or phases, per object.

1) A randomly selected object rotated on a table around its central vertical axis with a speed of $60^{\circ}$ per second for $8 \mathrm{~s}$, so that all sides of the object were seen.

2) Participants rated, by means of a slider on the screen, how well they recognized the object $(1=$ not well; $100=$ very well $)$. Then they typed the name of the object and indicated how certain they were of the name $(1=$ not certain, $100=$ very certain). If they did not know the name, they typed " 11 " and 
if they did not recognize the object, they typed " 00 " for the name of the object and the certainty ratings were ignored.

3) Participants selected the most appropriate category for the object from ten categories: clothes; cosmetics; cutlery and dishes; decoration; food; office supplies; tools; toys; kitchen utensils; unknown. They also rated how well the object fit the chosen category $(1=$ not at all; $100=$ very well $)$.

4) Participants rated how familiar the object was to them $(1=$ not familiar; $100=$ very familiar) and how detailed (visually complex $)$ it appeared to them $(1=$ not very detailed; $100=$ very detailed).

5) Participants rated how often they encounter the object at home ("contact") and how often they use the object at home (i.e., "usage"), also on scales from 1 (never) to 100 (very often).

Before the actual experiment, participants were presented with five practice scenes, in which one object (a pineapple) was rated, which is not part of OVO. The objects were presented in four blocks of 31 objects each. Between each block, participants were asked to take a break for 1 to $5 \mathrm{~min}$. The experiment took 75 min on average. Apart from the object in the practice trial, object presentation was randomized using the "Random.Range" function in Unity so that numbers (corresponding to individual objects) were chosen with equal probability from a finite set of numbers.

\section{Analysis \\ Descriptives}

The mean, standard deviation (SD), median, minimum, and maximum were calculated for each dimension (Recognizability, Familiarity, Details, Contact, Usage). Normality was tested with Shapiro-Wilk tests. Non-parametric (Spearman's rank) correlations were used to calculate correlation coefficients between the dimensions. Adjustment for multiple comparisons was implemented using Holm's method.

\section{Object Names}

For the naming data, we calculated the name agreement (NA, in $\%)$ and the $H$-statistic (Snodgrass and Vanderwart, 1980). The two categories of naming failures (" 11 " if they did not know the name, "00" if they did not recognize the object) were excluded from the analysis. Misspellings were included as the correctly spelled name and elaborations [e.g., halbes Brot ("half bread"), Haarkamm ("hairbrush")] were counted as separate names. If participants wrote down two distinct names for an object, only the first one counted. The NA is the percentage of people that produced the modal name (i.e., the name given by the majority of participants). The $H$-statistic is a different measure, which considers the frequency distribution of the given names as well as number of alternative names. An object that was given the same name from every participant in the sample would have an $H$-value of 0.00 . An object that elicited two different names with exactly the same frequency would have an $H$-value of 1.00 . That is, the larger the $H$-value, the smaller the name agreement. For a follow-up analysis, we additionally calculated the modal name per object and then grouped all words that literally contained the modal name before recalculating the $H$-statistic and the NA.
TABLE 1 | Summary statistics for all dimensions.

\begin{tabular}{|c|c|c|c|c|c|c|c|c|c|c|}
\hline & \multicolumn{2}{|c|}{ Recog } & \multicolumn{2}{|c|}{ Fam } & \multicolumn{2}{|c|}{ Details } & \multicolumn{2}{|c|}{ Contact } & \multicolumn{2}{|c|}{ Usage } \\
\hline & $Y$ & 0 & $Y$ & 0 & $Y$ & 0 & $Y$ & 0 & $Y$ & 0 \\
\hline M & 83.15 & 86.65 & 78.09 & 85.17 & 59.39 & 64.53 & 51.66 & 64.60 & 44.95 & 60.08 \\
\hline$S D$ & 17.64 & 14.41 & 14.73 & 14.92 & 18.14 & 16.32 & 24.09 & 25.97 & 24.66 & 26.88 \\
\hline Median & 90.44 & 91.88 & 79.94 & 89.12 & 62.71 & 68.20 & 54.71 & 69.64 & 42.74 & 60.08 \\
\hline Min & 25.03 & 22.12 & 29.85 & 16.64 & 15.18 & 14.16 & 2.24 & 2.24 & 1.06 & 1.12 \\
\hline Max & 99.68 & 98.36 & 99.50 & 99.64 & 86.11 & 91.00 & 95.06 & 99.16 & 94.47 & 99.60 \\
\hline
\end{tabular}

Y, Younger Adults; O, Older Adults; Recog, Recognizability; Fam, Familiarity.

\section{Object Categories}

For the semantic category analysis, we categorized the object based on which category was most often chosen by the participants (for the younger and older adults separately). Objects could only be assigned to one category. We calculated the number of objects that were attributed to the given category and the percentage of agreement (i.e., the percentage of participants that chose the objects in the category as belonging to that category). So, if a given category has two objects $\mathrm{A}$ and $\mathrm{B}$ and for object A $90 \%$ of participants said the items belonged to this category and for object B $60 \%$ put the item in this category, the average agreement for the category will be $75 \%$. Note that for some objects (e.g., apple), several versions were rated, which will increase the number of objects in that category (e.g., food).

\section{Comparison With Other Databases}

For comparison and to facilitate the pooling of objects, we provide the mean ratings across objects in our database together with those of two other 3D object (Peeters, 2018; Popic et al., 2020) and three colored-photograph databases (Adlington et al., 2009; Brodeur et al., 2010; Moreno-Martínez and Montoro, 2012). Common dimensions were the $H$-statistic $(H)$, the NA (in $\%)$, Familiarity, and Details. Since for Familiarity and Details, our objects were rated on a sliding scale from 1 to 100 and the other databases used a 5-point scale, we divided our scores by 20 . We acknowledge that rating on a scale from 1 to 100 is different from rating on a 5-point scale, thus we only report these measures and do not perform any statistical analyses.

\section{RESULTS}

The summary statistics for the collected norms (Recognizability, Familiarity, Details, Contact, and Usage) are presented in Table 1 for the younger and older adults separately. The Shapiro-Wilk tests showed that the data were not normally distributed for the younger nor for the older adults (all $p<0.05$ ). Figure 2 displays the ratings for each of the norms for the younger adults, the older adults, and the complete sample.

\section{Correlation Analysis}

To explore the relationships between the different dimensions, Spearman correlation analyses (Holm-corrected for multiple comparisons) were used (Table 2). For both samples, there were significant correlations between Contact and Usage, 


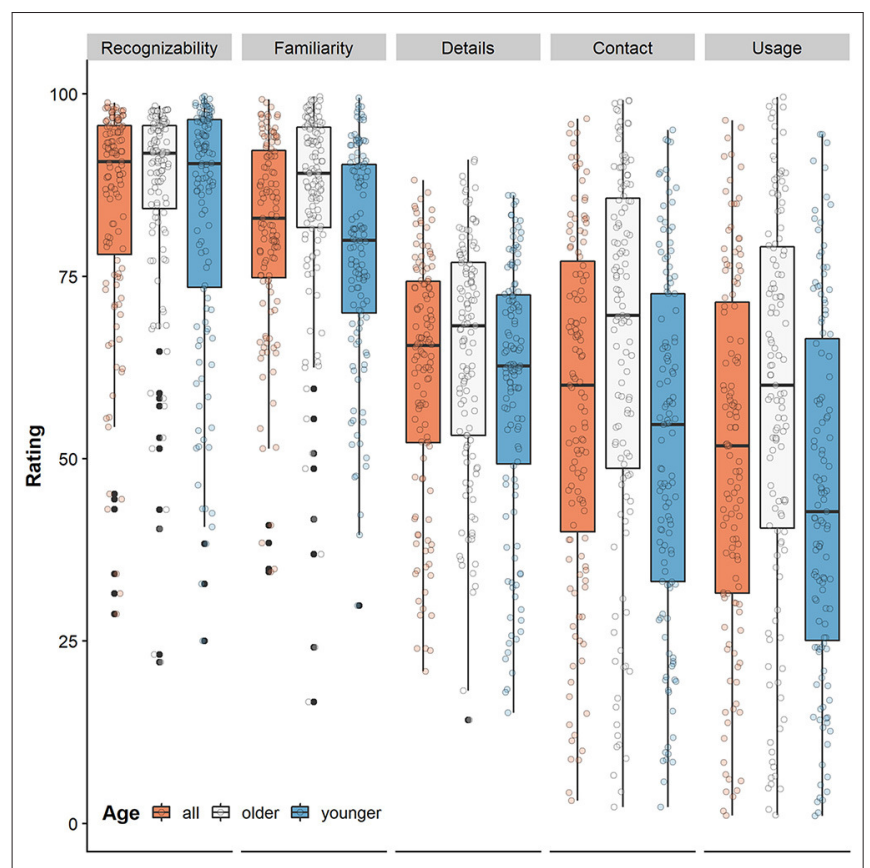

FIGURE 2 | Boxplots with individual data points for the object ratings for the entire sample (red) and per age group (older: white, younger: blue) per dimension $(1=$ not, $100=$ very). The horizontal line represents the median.

TABLE 2 | Correlation matrix for the younger (upper panel) and older (lower panel) adults.

\begin{tabular}{|c|c|c|c|c|c|}
\hline & Recog & Fam & Details & Contact & Usage \\
\hline Recog & 1.00 & & & & \\
\hline Fam & $0.69^{\star \star \star}$ & 1.00 & & & \\
\hline Details & $0.75^{\star \star \star}$ & $0.48^{* \star \star}$ & 1.00 & & \\
\hline Contact & 0.22 & $0.75^{\star \star \star}$ & 0.00 & 1.00 & \\
\hline Usage & 0.16 & $0.70^{\star \star \star}$ & -0.06 & $0.99^{\star \star \star}$ & 1.00 \\
\hline Recog & 1.00 & & & & \\
\hline Fam & $0.71^{\star \star \star}$ & 1.00 & & & \\
\hline Details & $0.76^{\star \star \star}$ & $0.56^{\star \star \star}$ & 1.00 & & \\
\hline Contact & $0.36^{\star \star \star}$ & $0.80^{* \star \star}$ & 0.15 & 1.00 & \\
\hline Usage & $0.31^{* \star}$ & $0.77^{\star \star \star}$ & 0.11 & $0.98^{\star \star \star}$ & 1.00 \\
\hline
\end{tabular}

Recog, Recognizability; Fam, Familiarity; ${ }^{*} p($ corr $)<0.05,{ }^{* \star} p$ (corr) $<0.01,{ }^{* \star *} p($ corr $)<$ 0.001 (multiple comparisons-corrected using Holm's method).

between Familiarity and Contact, and between Familiarity and Usage. Also, there was a positive correlation between Details and Recognizability for both age groups. For the older but not for the younger adults, Recognizability and Usage were significantly correlated.

\section{Object Names}

The percentage of "no recognition" responses (i.e., "I do not recognize the object," coded as " 00 ") was $2.56 \%$ for the younger and $2.47 \%$ for the older adults. The amount of "no name"
TABLE 3 | Distribution of objects over the categories for younger and older adults.

\begin{tabular}{lccccc}
\hline & \multicolumn{2}{c}{ Younger } & & \multicolumn{2}{c}{ Older } \\
\cline { 2 - 3 } \cline { 5 - 6 } Category & count & \% agree & & count & \% agree \\
\hline Clothes & 9 & 82.68 & & 8 & 80.50 \\
Cosmetics & 25 & 80.24 & & 23 & 82.56 \\
Decoration & 14 & 74.16 & & 15 & 72.00 \\
Dishes/cutlery & 8 & 69.85 & & 8 & 70.00 \\
Food & 18 & 95.92 & & 18 & 92.44 \\
Kitchen supplies & 12 & 69.36 & & 15 & 69.60 \\
Office supplies & 10 & 77.35 & 9 & 80.44 \\
Tools & 12 & 80.64 & 15 & 71.20 \\
Toys & 6 & 83.82 & 6 & 92.67 \\
Unknown & 9 & 43.14 & 6 & 47.33
\end{tabular}

responses (i.e., "I do not know the name of the object," coded as " 11 ") was $1.91 \%$ for the younger and $1.43 \%$ for the older adults.

The mean $H$-statistic for the younger adults was $1.86(S D=$ $1.09)$ and the average NA was $59.36 \%(S D=25.72 \%)$. For the older adult sample, the mean $H$-statistic was $2.12(S D=1.04)$ and the average NA was $52.02 \%(S D=24.31 \%)$. The $H$-statistic, modal names, NA, and the percentage of "no recognition" ("00") and "no name ("11") responses per object can be found in the online database (for the complete sample and separately for younger and older adults). In the follow-up analysis, grouping together all names that contained the modal name, the mean $H$ statistic was $1.28(S D=1.11)$ and the average NA was $72.76 \%$ (SD $=25.19 \%$ ) for the younger adults. For the older adult sample, the mean $H$-statistic was $1.60(S D=1.19)$ and the average NA was $63.98 \%(S D=27.64 \%)$.

\section{Object Categories}

Table 3 shows the distribution of objects over the semantic categories. "cosmetics" was the category with most objects and "toys" with the least objects for both age groups. The categorization of objects in the "toys" and "food" categories was the most consistent. Numerically, the younger adults categorized more objects as "unknown" than the older adults.

For each object, we provide the modal category, what percentage of participants chose this category (i.e., the percentage of agreement for this category), and which other categories were chosen. We also provide data per object on how well the objects fit the chosen category in the database online ( $1=$ not at all; 100 = very well).

\section{Object Categories}

Table 4 presents the comparison of ratings to other databases. The mean familiarity for OVO was numerically comparable to the norms of Brodeur et al. (2010) and slightly higher than the other databases (i.e., Adlington et al., 2009; MorenoMartínez and Montoro, 2012; Peeters, 2018; Popic et al., 2020). The average visual complexity as well as the $H$-statistic were higher for OVO compared to the other databases while the NA was lower. 
TABLE 4 | Overview of the standardized measures from the 3D objects in OVO (complete sample) and comparable databases.

\begin{tabular}{lccccc}
\hline & N & Fam & Details & $\boldsymbol{H}$ & NA (\%) \\
\hline OVO & 123 & 4.05 & 3.08 & 2.26 & 54.88 \\
Popic et al. (2020) & 121 & 4.37 & 2.42 & $/$ & 74.00 \\
Peeters (2018) & 147 & 3.20 & 2.69 & 1.05 & 74.99 \\
Moreno-Martínez and Montoro (2012) & 360 & 3.56 & 2.55 & 0.94 & 72.00 \\
Brodeur et al. (2010) & 480 & 4 & 2.4 & 1.65 & 64.00 \\
Adlington et al. (2009) & 147 & 3.76 & 2.89 & 1.11 & 67.61
\end{tabular}

Mean ratings for familiarity, details (visual complexity) and name agreement. $\mathrm{N}$, number of items; Fam, Familiarity; H, H-statistic; NA, name agreement.

\section{DISCUSSION}

To support VR-based research, assessment, and therapy, we created OpenVirtualObjects (OVO), a freely available set of 124 standardized 3D household objects, rated by healthy younger and older adults. In this paper, we provided summary statistics and correlation coefficients of these ratings as well as naming and sematic category information. We also compared a selection of the norms with those of five existing databases that include 3D objects or colored photographs (Adlington et al., 2009; Brodeur et al., 2010; Moreno-Martínez and Montoro, 2012; Peeters, 2018; Popic et al., 2020).

Recognizability ratings were generally high for both age groups, suggesting that the objects can be recognized well by both age groups. Familiarity ratings suggest that both groups were used to (most of) the objects. The ratings for contact and usage showed a larger variance, suggestion that OVO contains objects that people frequently encounter or use in their households and objects they do not encounter or use often.

Although we did not statistically compare them, it should still be noted that, numerically, older adults gave higher ratings than younger adults across the dimensions. It is difficult to disentangle whether this relates to a general effect of age group on the use of the rating scale or whether older adults really perceived the objects to be more recognizable, familiar, and visually complex than the younger adults-or, for contact and usage, whether older adults indeed come into contact and use the objects more frequently than younger adults. In other types of ratings, older adults were shown to give more extreme ratings than younger adults, for example for valence and arousal ratings of pictures (Grühn and Scheibe, 2008). In general, we advise OVO users to select objects based on the ratings from the age group that best resembles their target population.

The correlation analysis revealed that especially the dimensions contact and usage are highly correlated for both samples. This indicates that the items that participants often came into contact with, they also used often and vice versa, or that these dimensions measure the same item property. The positive correlation between details and recognizability suggests that objects with more details (i.e., higher visual complexity) were recognized better. This suggests for VR-based studies that the quality of the stimulus in VR, in our case the objects, is related to the recognizability of the object. Thus, for tasks that require the recognition of objects, researchers should aim to use as high-quality representations of VR objects as possible (e.g., given limitations in PC memory).

The category data revealed that most objects could be categorized within the pre-determined categories, which covered large parts of the household (e.g., kitchen supplies, tools, food, cosmetics). OVO users can pick and choose particular categories to use the objects with the highest norms relevant to their research goals. Of note, the category "toys" is particular, as it contains miniature versions of non-household objects (e.g., a train). Outside the (virtual) household setting or context, it might not be clear that these items are toys.

The database comparison showed that the familiarity ratings for OVO were comparable to or higher than for the other databases (Adlington et al., 2009; Brodeur et al., 2010; MorenoMartínez and Montoro, 2012; Peeters, 2018; Popic et al., 2020). This emphasizes the complementarity or additional usefulness of OVO. Visual complexity was rated higher in OVO than in the other databases. This could be because 3D objects are often more complex than 2D images. However, OVO visual complexity ratings were also higher than those in Peeters (2018) and in Popic et al. (2020), even though these were rated in immersive VR. Researchers and clinicians should take the complexity ratings into account, especially for studies in which the visual properties of the stimuli have to be strictly controlled. Future studies will have to address the differences in visual complexity ratings between $2 \mathrm{D}$ and $3 \mathrm{D}$ objects, and the properties that contribute to visual complexity of $3 \mathrm{D}$ objects. The $H$-statistic was higher and the NA was lower for OVO than for the other databases, suggesting less uniform naming behavior for the OVO objects. The strong variance in these statistics across OVO objects could be because, for some objects, participants entered detailed descriptions with only fine-grained deviations [e.g., mechanischer Wecker ("mechanical alarm clock") and Analogwecker ("analog alarm clock")]. To facilitate the comparison with the other databases, we treated these elaborations as distinct names in our main analysis. However, such strict conventions may not be necessary or appropriate for all studies using the OVO objects. In a followup analysis, we grouped all names that literally contain the modal name (i.e., summarized all elaborations under the modal name) and found that the resulting $H$-statistics and NA were in the range of those reported for other databases. For many studies such an account should be sufficient to ensure that an object was recognized as belonging to the concept described by the modal name. For researchers that require exact naming distinctions and high levels of name agreement, we suggest to filter the OVO database by defining a threshold for the $H$-statistic or the percentage of name agreement. We provide data and $\mathrm{R}$ script templates to perform such operations.

In sum, we provide a set of $3 \mathrm{D}$ objects that are recognizable and familiar to both older and younger adults. Our set includes a variety of validated objects that are often encountered and used in a household setting. Various categories of household objects are represented in the database and the familiarity scores are comparable to those of other databases. We invite researchers 
and clinicians to choose their own set of objects based on the norms, age of the rating group, and other properties that are most relevant for their purposes. A concrete example for the use of $\mathrm{OVO}$ is to use the objects for a spatial memory assessment task in VR, like the virtual memory task (VMT) as described by Koenig et al. (2011). In the VMT, household items are presented on a virtual table and the participant or patient is asked to memorize the location of the objects. Then, the objects disappear and the participants is asked to recall the location of each object. The number of items to be memorized is gradually increased from four to seven and also perspective changes can occur during the task (i.e., the participant has to recall the object locations from a different side of the table). One of the important outcome measures is the difference between the actual location and the recall location (displacement error). If OVO objects were to be used in this type of study, one could select certain objects (i.e., those with similar ratings) to reduce the influence of undesirable object characteristics such as familiarity, visual complexity and name agreement on the memory process that is of interest. Another possibility is to include the ratings in the statistical analyses to account for their influence on the outcome measure. Finally, OVO provides a handle to select objects in advance or to account for differences in the perception and memory retrieval of objects and their names based on age differences. We provide all scripts of the analyses described in this paper in the online database, so that users can easily tailor the analyses to their needs and select the most appropriate set of objects or ratings.

Although this database can provide a step forward in the standardization and optimization of VR-based experimentation, it also has limitations. The rating study was conducted with German participants, so the data might not be representative for other cultural settings (e.g., non-European). Also, it was done in $2 \mathrm{D}$ on a computer screen rather than in a fully immersive VR environment. Thus, although the objects were $3 \mathrm{D}$ representations (and not pictures), they were presented (rotating) in 2D. We chose this procedure for pragmatic reasons, namely to increase the number of participants while still collecting all norms for all objects from each participant. Furthermore, we wanted to ensure that the norms are not limited to a specific VR headset (e.g., the Oculus Rift or the HTC Vive). We also did not systematically test for usability of the objects in interactive, real-time applications. Some of the rated objects have a high number of vertices, which increases the level of detail and the naturalistic look of the objects but might lead to computational challenges in interactive and real-time scenarios. This will, to a great extent, depend on the specific implementation and the hardware used and should be tested during early stages of task development. As a first step to alleviate this challenge, we also provide "low-poly" versions (< 10,000 vertices) of all the objects. It should be noted that these were not part of the participant ratings we present above and that, despite the lowered vertex counts, these objects might still be too graphically expensive for some tasks and hardware setups. For future research, it would be beneficial to collect more norms using immersive and interactive VR or to explicitly compare screen and HMD norms as well as high- and low-poly versions of the same objects. These studies could be conducted with the Unity code and the object versions provided in the database.
Another limitation is that, in contrast to Peeters (2018), we did not collect image agreement scores (i.e., scores of how well the object fits the modal name it was given). Again, this was a pragmatic decision to reduce experiment time and these norms can be easily collected with the existing experiment code, by providing the modal names collected in OVO together with the objects, to a new group of participants.

\section{CONCLUSION}

VR provides new opportunities for researchers and clinicians to create realistic yet controlled experiments and applications. However, it remains expensive and time-consuming to develop and standardize VR content, which is necessary for reliable and replicable results obtained in VR. In this paper, we presented OVO, a freely available set of 124 realistic $3 \mathrm{D}$ household objects for VR-based testing, training, clinical diagnostics, and rehabilitation. Based on ratings by healthy younger and older adults, we provide norms of recognizability, familiarity, visual complexity, contact, and usage in daily life. We also report naming and category information. All objects, data, and scripts (analysis and testing) are freely available online (https://edmond. mpdl.mpg.de/imeji/collection/7L7t07UXD8asG_MI). We hope that the objects in this database are useful in experimental, educational, and clinical settings-or in other situations that require standardized $3 \mathrm{D}$ stimuli. We invite researchers to select objects according to their research questions and target populations and to add more objects or norm values to the database whenever possible. By creating and sharing highquality, freely available VR content, we can increase the amount and quality of VR-based experimental research and clinical applications.

\section{DATA AVAILABILITY STATEMENT}

The datasets generated for this study can be found in online repositories. The names of the repository/repositories and accession number(s) can be found at: https://edmond.mpdl.mpg. de/imeji/collection/7L7t07UXD8asG_MI\&lt.

\section{ETHICS STATEMENT}

The studies involving human participants were reviewed and approved by Psychology Department at the HumboldtUniversität, Berlin. The patients/participants provided their written informed consent to participate in this study.

\section{AUTHOR CONTRIBUTIONS}

JT, FK, SK, MA, EQ, and JB looked for objects. JT, FK, SK, MG, EQ, and JB designed the experiment. LP and MA programmed and conducted the experiment. The analysis was done by JT, FK, MG, and LP analysis. JT, FK, and MG wrote the manuscript. All authors contributed to the article and approved the submitted version. 


\section{FUNDING}

This project was funded by a grant (BMBF grant 13GW0206) from the German Federal Ministry for Education and Research for the research consortium VReha-Virtual worlds for digital diagnostics and cognitive rehabilitation.

\section{REFERENCES}

Adlington, R. L., Laws, K. R., and Gale, T. M. (2009). The hatfield image test (HIT): a new picture test and norms for experimental and clinical use. J. Clin. Exp. Neuropsychol. 31, 731-753. doi: 10.1080/13803390802488103

Aukstakalnis, S., and Blatner, D. (1992). Silicon Mirage; The Art and Science of Virtual Reality. Berkeley, CA: Peachpit Press.

Brady, T. F., Störmer, V. S., and Alvarez, G. A. (2016). Working memory is not fixed-capacity: More active storage capacity for real-world objects than for simple stimuli. Proc. Natl. Acad. Sci. U.S.A. 113, 7459-7464. doi: 10.1073/pnas.1520027113

Brodeur, M. B., Dionne-Dostie, E., Montreuil, T., and Lepage, M. (2010). The Bank of Standardized Stimuli (BOSS), a new set of 480 normative photos of objects to be used as visual stimuli in cognitive research. PLoS ONE 5:e10773. doi: 10.1371/journal.pone.0010773

Frisch, S., Förstl, S., Legler, A., Schöpe, S., and Goebel, H. (2012). The interleaving of actions in everyday life multitasking demands. J. Neuropsychol. 6, 257-269. doi: 10.1111/j.1748-6653.2012.02026.x

Grühn, D., and Scheibe, S. (2008). Age-related differences in valence and arousal ratings of pictures from the International Affective Picture System (IAPS): do ratings become more extreme with age? Behav. Res. Methods 40, 512-521. doi: 10.3758/BRM.40.2.512

Howard, M. C. (2017). A meta-analysis and systematic literature review of virtual reality rehabilitation programs. Comp. Hum. Behav. 70, 317-327. doi: 10.1016/j.chb.2017.01.013

Ioannidis, J. P. A. (2005). Why most published research findings are false. PLoS Med. 2:e124. doi: 10.1371/journal.pmed.0020124

Josman, N., Klinger, E., and Kizony, R. (2008). "Performance within the virtual action planning supermarket (VAP-S): An executive function profile of three different populations suffering from deficits in the central nervous system," in 7th International Conference on Disability Virtual Reality and Associated Technologies with ArtAbilitation (Maia), 33-38.

Klotzsche, F., Mariola, A., Hofmann, S., Nikulin, V. V., Villringer, A., and Gaebler, M. (2018). "Using EEG to decode subjective levels of emotional arousal during an immersive VR roller coaster ride," in 2018 IEEE Conference on Virtual Reality and 3D User Interfaces (VR) (Reutlingen), 605-606. doi: 10.1109/VR.2018.8446275

Koenig, S., Crucian, G. P., Dünser, A., Bartneck, C., and Dalrymple-Alford, J. C. (2011). Validity evaluation of a spatial memory task in virtual environments. Int. J. Des. Innov. Res. 6, 1-13.

Krohn, S., Tromp, J., Quinque, E. M., Belger, J., Klotzsche, F., Rekers, S., et al. (2020). Multidimensional evaluation of virtual reality paradigms in clinical neuropsychology: application of the VR-check framework. J. Med. Internet Res. 22:e16724. doi: $10.2196 / 16724$

Moreno-Martínez, F. J., and Montoro, P. R. (2012). An ecological alternative to snodgrass \& vanderwart: 360 high quality colour images with norms for seven psycholinguistic variables. PLoS ONE 7:e37527. doi: 10.1371 /journal.pone.0037527

Morina, N., Ijntema, H., Meyerbröker, K., and Emmelkamp, P. M. G. (2015). Can virtual reality exposure therapy gains be generalized to real-life? a metaanalysis of studies applying behavioral assessments. Behav. Res. Therapy 74 , 18-24. doi: 10.1016/j.brat.2015.08.010

\section{ACKNOWLEDGMENTS}

We would like to thank all members of the VReha team, including Paul Chojecki, Cade McCall, Carsten Finke, and Angelika Thöne-Otto for their valuable input during the meetings of the VReha-Virtual worlds for digital diagnostics and cognitive rehabilitation consortium.

Osterrieth, P. A. (1944). Le test de copie d'une figure complexe; contribution à l'étude de la perception et de la mémoire. [Test of copying a complex figure; contribution to the study of perception and memory]. Arch Psychol. 30, 206-356.

Peeters, D. (2018). A standardized set of 3-D objects for virtual reality research and applications. Behav. Res. Methods 50, 1047-1054. doi: 10.3758/s13428-017-0925-3

Popic, D., Pacozzi, S. G., and Martarelli, C. S. (2020). Database of virtual objects to be used in psychological research. PLOS ONE 15:e0238041. doi: 10.1371/journal.pone.0238041

Rey, A. (1941). L'examen psychologique dans les cas d'encéphalopathie traumatique. (Les problems.). [The psychological examination in cases of traumatic encepholopathy problems.]. Arch. Psychol. 28, 215-285.

Rizzo, A., Difede, J., Rothbaum, B. O., Reger, G., Spitalnick, J., Cukor, J., et al. (2010). Development and early evaluation of the virtual Iraq/Afghanistan exposure therapy system for combat-related PTSD. Ann. N. Y. Acad. Sci. 1208, 114-125. doi: 10.1111/j.1749-6632.2010. 05755.x

Rizzo, A., and Koenig, S. T. (2017). Is clinical virtual reality ready for primetime? Neuropsychology, 31, 877-899. doi: 10.1037/neu0000405

Simmons, J. P., Nelson, L. D., and Simonsohn, U. (2011). False-positive psychology: undisclosed flexibility in data collection and analysis allows presenting anything as significant. Psychol. Sci. 22, 1359-1366. doi: $10.1177 / 0956797611417632$

Singh, A., Sha, J., Narayan, K. S., Achim, T., and Abbeel, P. (2014). "BigBIRD: a large-scale 3D database of object instances," in 2014 IEEE International Conference on Robotics and Automation (ICRA) (Hong Kong), 509-516. doi: 10.1109/ICRA.2014.6906903

Słyk, S., Zarzycki, M. Z., Kocwa-Karnaś, A., and Domitrz, I. (2019). Virtual reality in the diagnostics and therapy of neurological diseases. Exp. Rev. Med. Devices 16, 1035-1040. doi: 10.1080/17434440.2019.16 93892

Snodgrass, J. G., and Vanderwart, M. (1980). A standardized set of 260 pictures: Norms for name agreement, image agreement, familiarity, and visual complexity. J. Exp. Physchol. Hum. Learn. 6, 174-215. doi: 10.1037/0278-7393.6.2.174

Tromp, J., Peeters, D., Meyer, A. S., and Hagoort, P. (2018). The combined use of virtual reality and EEG to study language processing in naturalistic environments. Behav. Res. Methods 50, 862-869. doi: 10.3758/s13428-017-0911-9

Conflict of Interest: The authors declare that the research was conducted in the absence of any commercial or financial relationships that could be construed as a potential conflict of interest.

Copyright (c) 2020 Tromp, Klotzsche, Krohn, Akbal, Pohl, Quinque, Belger, Villringer and Gaebler. This is an open-access article distributed under the terms of the Creative Commons Attribution License (CC BY). The use, distribution or reproduction in other forums is permitted, provided the original author(s) and the copyright owner(s) are credited and that the original publication in this journal is cited, in accordance with accepted academic practice. No use, distribution or reproduction is permitted which does not comply with these terms. 